\title{
A structured inventory of spiders (Arachnida, Araneae) in natural and artificial forest gaps at Porto Urucu, Western Brazilian Amazonia
}

\author{
Alexandre B. BONALDO ${ }^{1}$, Sidclay C. DIAS ${ }^{2}$
}

\begin{abstract}
A preliminary survey of the spider fauna in natural and artificial forest gap formations at "Porto Urucu", a petroleum/natural gas production facility in the Urucu river basin, Coari, Amazonas, Brazil is presented. Sampling was conducted both occasionally and using a protocol composed of a suite of techniques: beating trays (32 samples), nocturnal manual samplings (48), sweeping nets (16), Winkler extractors (24), and pitfall traps (120). A total of 4201 spiders, belonging to 43 families and 393 morphospecies, were collected during the dry season, in July, 2003. Excluding the occasional samples, the observed richness was 357 species. In a performance test of seven species richness estimators, the Incidence Based Coverage Estimator (ICE) was the best fit estimator, with 639 estimated species. To evaluate differences in species richness associated with natural and artificial gaps, samples from between the center of the gaps up to 300 meters inside the adjacent forest matrix were compared through the inspection of the confidence intervals of individual-based rarefaction curves for each treatment. The observed species richness was significantly higher in natural gaps combined with adjacent forest than in the artificial gaps combined with adjacent forest. Moreover, a community similarity analysis between the fauna collected under both treatments demonstrated that there were considerable differences in species composition. The significantly higher abundance of Lycosidae in artificial gap forest is explained by the presence of herbaceous vegetation in the gaps themselves. Ctenidae was significantly more abundant in the natural gap forest, probable due to the increase of shelter availability provided by the fallen trees in the gaps themselves. Both families are identified as potential indicators of environmental change related to the establishment or recovery of artificial gaps in the study area.
\end{abstract}

KEYWORDS: spider inventories, richness estimators, forest gaps, Brazilian Amazonia.

\section{Inventário estruturado de aranhas (Arachnida, Araneae) em clareiras naturais e artificiais em Porto Urucu, Amazônia Ocidental Brasileira}

\section{RESUMO}

Apresenta-se um inventário preliminar da fauna de aranhas em clareiras naturais e artificiais em "Porto Urucu", uma instalação produtora de petróleo e gás natural na bacia do Rio Urucu, Coari, Amazonas, Brasil. As amostras foram realizadas de modo ocasional e com um protocolo estruturado composto por um conjunto de técnicas de amostragem: guarda-chuvas entomológicos (32 amostras), amostras manuais noturnas (48), rede de varredura (16), extratores de Winkler (24) e armadilhas de queda (120). Um total de 4201 aranhas pertencentes a 43 famílias e 393 morfoespécies foi coletado durante a estação seca em julho de 2003. Excluindo as amostras ocasionais, a riqueza observada foi de 357 espécies. No teste de desempenho de sete estimadores, ICE (Incidence Based Coverage Estimator) gerou o melhor resultado, com 639 espécies estimadas. Para avaliar diferenças na riqueza de espécies associadas a clareiras naturais e artificiais, conjuntos de amostras do centro da clareira a até $300 \mathrm{~m}$ adentro da floresta adjacente foram comparados através da inspeção dos intervalos de confiança de curvas de rarefaçáo baseadas no número de indivíduos de cada tratamento. A riqueza observada foi significantemente maior nas clareiras naturais combinadas com as florestas adjacentes do que nas clareiras artificiais combinadas com as matas do entorno. Além disso, uma análise de similaridade entre as faunas coletadas em ambos os tratamentos mostrou que existe diferenças consideráveis na composição de espécies. A abundância significativamente mais alta de Lycosidae nas florestas de clareiras artificiais é explicada pela presença de vegetação herbácea nas clareiras propriamente ditas. Ctenidae foi significantemente mais abundante nas florestas de clareiras naturais, provavelmente devido ao aumento da disponibilidade de abrigos causado pela queda de árvores nas clareiras propriamente ditas. Ambas estas famílias são identificadas como potenciais indicadores de mudanças ambientais relacionadas com o estabelecimento ou recuperação de clareiras artificiais na área de estudo.

PALAVRAS-CHAVE: inventários de aranhas, estimadores de riqueza, clareiras, Amazônia Brasileira.

${ }_{1}^{1}$ Museu Paraense Emílio Goeldi, E-mail: bonaldo@museu-goeldi.br

${ }^{2}$ Museu Paraense Emílio Goeldi, E-mail: pachistopelma@hotmail.com 


\section{INTRODUCTION}

Structured inventories of spider communities have been implemented on a regular basis in Amazon Basin only recently (Silva, 1992, 1996). Despite the fact that several such initiatives have been conducted in Brazilian Amazonia during the last 10 years, only two papers dealing with spider community structure have been published so far. Borges \& Brescovit (1996) provided data from two western Amazonian localities, The "Reserva de Desenvolvimento Sustentado Mamirauá" and "Boca do Sumaúma", both near Tefé, State of Amazonas. Höfer \& Brescovit (2001) presented results of a long term spider inventory carried out at the "Reserva Florestal Adolfo Ducke" (RFAD), Manaus, Amazonas.

Porto Urucu, the locality addressed in this paper, is practically unknown regarding the composition of its spider communities. Only six spider species from that area were recorded in the literature so far. The Ctenids Centroctenus auberti (Caporiacco), Ctenus inaja Hofer, Brescovit \& Gasnier, Ctenus villasboasi Mello-Leitão, Cupiennius celerrimus Simon and Ancylometes rufus (Walckenaer) were reported by Brescovit (1996), Höfer et al. (1994), Brescovit \& Eickstedt (1995) and Höfer \& Brescovit (2000). A single species has Porto Urucu as its type locality, Rhoicinus urucu (Trechaleidae), described by Brescovit \& Oliveira (1994) on specimens collected occasionally in bromeliads during a floristic and herpetological inventory carried out in early 1990s.

The study area is located nearly 600 kilometers southwest Manaus and harbors an oil/gas exploitation facility established in the 1980s. One of the most conspicuous results of this intervention is the creation of deforestation gaps in the dry land forest matrix to allow activities related to probing, establishing and operating petroleum and gas wells. These gaps are opened for several specific purposes, such as helicopter landing, drilling of wells and assembling of equipment for $\mathrm{oil} /$ gas extraction and transferring. One of the most common kinds of artificial gaps in Porto Urucu is the so called "Jazidas" (here termed JAZ gaps), represented by the gaps assigned for the aerial deposit of soil and raw materials for highway construction, typically located near the margins of the roads. These clear-cut, soil-compacted areas are probably the most distinct artificial gaps in the study area.

In order to access the spider assemblages in both natural and artificial forest gaps, as well as in the dry land forest matrix adjacent to those gaps, we compared a set of high impacted JAZ gaps, each one from five to seven years in age, and a set of natural forest gaps, formed by the natural fall of old trees. This paper reports a first attempt to describe the spider communities under these novel environmental conditions and provides the following preliminary data on the Urucu spider community structure as well as on the impact of oil/ gas exploitation activities in the spider fauna of the study area:
(1) a general checklist, including data on species composition obtained both occasionally and using a structured inventory; (2) a characterization of the differences in species richness and composition of the spider fauna present in both artificial and natural forest gaps formations; (3) comparisons between the performance of several richness species estimators and; (4) suggestions of appropriate environmental indicator taxa for both establishment and recovery of artificial gaps in the study area.

\section{MATERIAL AND METHODS}

\section{STUDY AREA}

"Porto Urucu" is a 54000 ha petroleum/natural gas production unity in the right margin of Urucu River, Solimones river basin, State of Amazonas, Brazil ( $\left.4^{\circ} 53^{\prime} \mathrm{S}, 65^{\circ} 20^{\prime} \mathrm{W}\right)$. Regional climate is classified as "Af" by the Köppen system, with the rainy season between September and April, and the dry season between May and August (RADAM-BRASIL, 1978). The main forest formation in Porto Urucu is "terra firme" (dry land) associated with lowlands and plateaus as described by Ribeiro et al. (1999) and cut by small creeks ("Igarapés"), tributaries of the Urucu River. Three main creeks are located in the study area (Lontra, Tartaruga and Marta creeks). Natural gaps are formations produced in the forest matrix from fallen trees or large branches from the canopy, exposing the forest ground to direct solar radiation. The natural forest gaps sampled were coded as NAT1 (040 51'17.7' S, 65 04'14.4”W), NAT2 (04 51'22.5” S, 65 04'16.2”W),

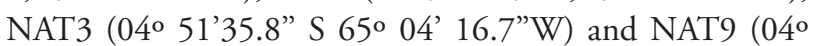
$53^{\prime} 42^{\prime \prime}$, 65 $15^{\circ} 18^{\prime \prime W)}$. All artificial forest gaps sampled were originated from the construction or maintenance of the Porto Urucu road network, named JAZ 19 (04'52'07"S,

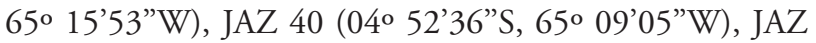

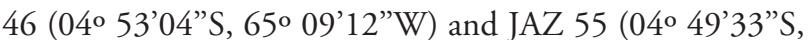
$\left.65^{\circ} 01^{\prime} 50^{\prime \prime} \mathrm{W}\right)$.

\section{COLLECTING}

The data presented here were obtained in a single expedition carried out in July, 2003. Five methods were used to access the spider fauna; pitfall traps, Winkler extractors, beating trays, sweep-net and nocturnal hand searches, during 13 days by three collectors. The samples were obtained from both the center and the edge of a given forest gap, as well as inside the forest immediately associated with the gap, until 300 meters from gap edge. The edge of a given forest gap was roughly defined as a ten meters wide band circling the gap. Sweeping was performed only in the center of each forest gap and the nocturnal hand search was not applied in the gap edges. There was a total of 120 pitfall trap samples (60 in the natural forests gap and 60 in the artificial forests gap), 
24 Winkler extractor samples (three by area), 32 beating tray samples, 16 samples of sweep-net and 48 samples of nocturnal collections, were obtained. Pitfall traps were used to access the litter-dwelling spiders. Each trap, composed of a $500 \mathrm{ml}$ plastic cup of $10 \mathrm{~cm}$ diameter buried to the level of the soil, was placed at least 5 meters apart one from another. Alcohol $\left(80^{\circ}\right.$ proof) was used as the preserving liquid.

Table 1 - Sampling design applied to the structured inventory of spiders in natural and artificial forest gap areas in Porto Urucu, Coari, Amazonas, Brazil.

\begin{tabular}{lccccccc}
\hline \multirow{2}{*}{ Method } & \multicolumn{2}{c}{ Gap } & \multicolumn{2}{c}{ Edge } & \multicolumn{4}{c}{$\begin{array}{c}\text { Adjacent } \\
\text { forest }\end{array}$} & Total \\
\cline { 2 - 7 } & NAT & JAZ & NAT & JAZ & NAT & JAZ & \\
\hline Pitfall-trap & 20 & 20 & 20 & 20 & 20 & 20 & 120 \\
Winkler extractor & 4 & 4 & 4 & 4 & 4 & 4 & 24 \\
Beating tray & - & - & 4 & 4 & 12 & 12 & 32 \\
Sweep-net & 8 & 8 & - & - & - & - & 16 \\
$\begin{array}{l}\text { Nocturnal hand } \\
\text { search }\end{array}$ & 12 & 12 & - & - & 12 & 12 & 48 \\
Total & 44 & 44 & 28 & 28 & 48 & 48 & 240 \\
\hline
\end{tabular}

Beating trays were used to access shrub-dwelling spiders during the day. Each beating tray was comprised of a $0,80 \mathrm{~m}^{2}$ cotton white sheet. Nocturnal hand searches were modified from the "looking up - looking down" method proposed by Coddington et al. (1991). More detailed descriptions of these methods are avaliable in Coddington et al. (1991) and Brescovit et al. $(2002,2004)$. The samples combined across each methodology were the results of: one pitfall trap exposed by 10 days, one square meter litter (sifted and included in Winkler extractors for 3 days), one hour of beating per collector, one hour of sweeping per collector and, one hour of nocturnal hand search per collector, inside an area of $300 \mathrm{~m}^{2}$. To improve the taxonomic list of the study area, we also performed occasional samplings in the margins of the Lontra and Tartaruga creeks. These occasional samplings were carried out using time-independent foliage beating during the day and time and area-free hand searching at night. Voucher specimens were deposited in the arachnological collections of Museu Paraense Emílio Goeldi, Belém (MPEG).

\section{STATISTICAL PROCEDURES}

Sample intensity (SI) was defined as the ratio of adults to species (Coddington et al., 1996). Seven non-parametric estimators of species richness (Chao 1, 2, Jackknife 1, 2, Bootstrap, ACE and ICE) were used in order to verify which richness estimators fit best to the present data set. Detailed descriptions of these algorithms are available in Colwell \& Coddington (1994), Toti et al. (2000) and Colwell (2006). To reduce the influence of the sample order, 100 randomizations of sample sequence were made for each sample increment (Coddington et al. 1996). This analysis was performed using
EstimateS software 7.5 (Colwell, 2006). Differences in species richness in both JAZ and NAT communities (defined as those under the influence of either artificial or naturals forest gaps) were visualized through the inspection of the $95 \%$ confidence intervals of individual-based rarefaction curves calculated using EcoSim 7 (Gotelli \& Entsminger, 2001). Kulczynski's (sensu Toti et al., 2000) and SØrensen's index of similarity were used to compare the composition of species in the two communities (Wolda, 1981; Krebs, 1999). To test the normality of the abundance data of Ctenidae and Lycosidae using the total sum of individuals (juveniles and adults) present in each JAZ and NAT samples, the Lilliefors test was implemented in BioEstat 4.0 (Ayres et al., 2005). As the Ctenidae data set was homocedastic and the Lycosidae one was heterocedastic, Student's T test and Mann-Whitney tests were used respectively, also through BioEstat 4.0 , to verify the differences in abundance of these families in both artificial and natural gap forests.

\section{RESULTS AND DISCUSSIONS}

\section{ASSEMBLAGE COMPOSITION}

The samples obtained in Porto Urucu from both natural and artificial forest gaps, together with those obtained occasionally in the vicinities of the Lontra and Tartaruga creeks, produced 4201 spiders, belonging to 43 families. The most abundant family was Ctenidae, with 714 specimens, followed by Araneidae, Lycosidae, Theridiidae and Salticidae, each one accounting for between 400 to 500 individuals. These five families represented 58.3\% of all spiders collected. Seven families were recorded by only one specimen (Clubionidae, Dictynidae, Hahniidae, Idiopidae, Nephilidae, Paratropididae and Tetrablemmidae) and three were represented by two specimens each (Cyrtaucheniidae, Hersiliidae and Palpimanidae) (see Fig. 1 for data on abundance per family).

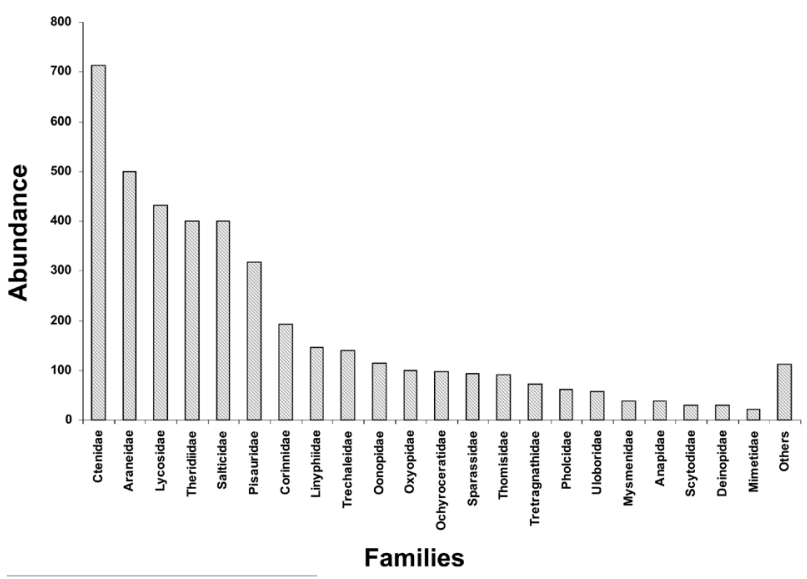

Figure 1 - Number of individuals per family collected in Porto Urucu, Coari, Amazonas, Brazil. Families with less than 20 individuals were grouped as "others". 
Considering only the adult spiders collected, the number of families represented in the samples decreases to 37, as Idiopidae, Paratropididae, Tetrablemmidae (represented by singletons), Cyrtaucheniidae (two individuals), Gnaphosidae (five) and Zodariidae (11) were represented only by unidentifiable juveniles. The observed richness was 393 species or morphospecies, represented by 1612 adults $(38.3 \%$ of all spiders collected). From all the recognized species, only 47 were identified to species level, highlighting the poor taxonomic knowledge of the Western Brazilian Amazonia spiders. Only four of these species were previously reported to occur in Porto Urucu. Two remaining species reported to the study area in the taxonomic literature, Cupiennius celerrimus and Rhoicinus urucu, were not re-collected.

The sampling intensity was only four individuals per species, a low value when compared with the figures obtained in structured inventories in North America (e.g. Coddington et al., 1996, Appalachian Mountains, SI = 18.3) and Europe (e.g. Scharff et al., 2003, Denmark, SI = 132). However, low sampling intensity values are common in inventories carried out in tropical sites, such as those reported by Coddington et al. (1991) (Bolivia, three localities, SI < 5), and even in some sub-tropical sites, as reported by Bonaldo et al. (2007) (State of Rio Grande do Sul, Brazil; SI = 10.4, but inflated by the inclusion of identifiable juveniles in the calculation; $\mathrm{SI}=8.8$, excluding the identifiable juveniles and few species identified only by juveniles). The number of species represented by singletons was 167 . Thus, the inventory completeness, defined by Coddington et al. (1996) as the percentage of species represented by singletons, is still high ( $42.5 \%$ of all recognized species). This is another indication that this preliminary inventory is far from complete.

In terms of number of species, the richer families were Theridiidae (68 spp.), Salticidae (60 spp.) and Araneidae (57 spp.), confirming the general pattern observed in Neotropical ecosystems (Silva, 1992). Table 2 depicts the species and morphospecies recorded in Porto Urucu, with the number of individuals collected by sampled area. The occasional sampling in the Igarapés Marta and Tartaruga added 36 species to the list, mainly representatives of Pholcidae and Trechaleidae. At least some of these species are characteristically found near water, as in the case of several Trechaleidae and some Pisauridae (Höfer \& Brescovit, 2001).

Table 2 - List of spider species and morphospecies recorded from Porto Urucu, Coari, Amazonas, Brazil, with the number of adults collected in each sampling site. NAT, natural gaps; JAZ, artificial gaps; IGL, Igarapé (creek) Lontra; IGT, Igarapé (creek) Tartaruga.

\begin{tabular}{|c|c|c|c|c|c|c|c|c|c|c|c|}
\hline Species/Areas & N1 & $\mathrm{N} 2$ & N 3 & N9 & $\mathrm{J} 19$ & $\mathrm{~J} 40$ & $\mathrm{~J} 46$ & $\mathrm{~J} 55$ & IGL & IGT & TOTAL \\
\hline \multicolumn{12}{|l|}{ Anapidae } \\
\hline Gen. sp. 1 & & 5 & 1 & & & 1 & & & & & 7 \\
\hline Gen. sp. 2 & & & & & 2 & & & 2 & & & 4 \\
\hline Gen. sp. 3 & & 4 & 2 & & 1 & & & & & & 7 \\
\hline Gen. sp. 4 & & & & & & & & 1 & & & 1 \\
\hline Anapisona sp. 1 & & & & 2 & & & & 1 & & & 3 \\
\hline \multicolumn{12}{|l|}{ Anyphaenidae } \\
\hline Gen. sp. 1 & & & 1 & & & & & & & & 1 \\
\hline \multicolumn{12}{|l|}{ Araneidae } \\
\hline Gen. sp. 1 & & & & & & & & & 1 & & 1 \\
\hline Gen. sp. 2 & & & & & 2 & & & & & & 2 \\
\hline Gen. sp. 3 & & & & & 2 & & & & & & 2 \\
\hline Gen. sp. 4 & & & & & & 1 & & & 1 & & 2 \\
\hline Acacesia sp. 1 & 1 & & 1 & & & & 1 & & & & 3 \\
\hline Alpaida aff. antonio & 4 & & 1 & 1 & & & 2 & & & & 8 \\
\hline Alpaida bicornuta & & & & & & & 1 & & & & 1 \\
\hline Alpaida delicata & 1 & & 6 & & 8 & & 4 & 8 & 3 & 2 & 32 \\
\hline Alpaida leucogramma & & & & & 4 & 1 & 1 & 2 & & & 8 \\
\hline Alpaida sp. 1 & & 2 & 1 & & 3 & 5 & 1 & 2 & & & 14 \\
\hline Alpaida sp. 2 & & & & 1 & & & & & & & 1 \\
\hline Alpaida sp. 3 & & & & & & & 1 & & & 1 & 2 \\
\hline Alpaida sp. 4 & & & & & & 1 & & & & & 1 \\
\hline Alpaida sp. 5 & & & & & & & & & & 2 & 2 \\
\hline Alpaida sp. 6 & 4 & & & & & & & & & & 4 \\
\hline
\end{tabular}


ACTA

AMAZONICA

A structured inventory of spiders (Arachnida, Araneae) in natural and artificial forest gaps at Porto Urucu, Western Brazilian Amazonia

Table 2 - Continuação

\begin{tabular}{|c|c|c|c|c|c|c|c|c|c|c|c|}
\hline Species/Areas & N 1 & $\mathrm{~N} 2$ & N 3 & N9 & $\mathrm{J} 19$ & $\mathrm{~J} 40$ & $\mathrm{~J} 46$ & $\mathrm{~J} 55$ & IGL & IGT & TOTAL \\
\hline Alpaida sp. 7 & & & & & & 1 & & & & & 1 \\
\hline Alpaida sp. 8 & & & & & & & & 1 & & & 1 \\
\hline Alpaida sp. 9 & & & & & 1 & & & & & & 1 \\
\hline Alpaida sp. 10 & & & & & 1 & & & & & & 1 \\
\hline Alpaida sp. 11 & & & & & & & & & 1 & 1 & 2 \\
\hline Alpaida sp. 12 & & & & & & & 1 & & & & 1 \\
\hline Argiope argentata & & & & & & & 6 & & & & 6 \\
\hline Aspidolasius sp. 1 & & & 1 & & & & & & & & 1 \\
\hline Azilia sp. 1 & 1 & & & & & & & & & & 1 \\
\hline Chaetacis aureola & 1 & & & & 1 & & & & & & 2 \\
\hline Cyclosa fililineata & & 1 & & & & 1 & & & & & 2 \\
\hline Cyclosa longicauda & & & & & 1 & & & & & & 1 \\
\hline Eustala sp. 1 & & 1 & 2 & & 2 & & & & 2 & 1 & 8 \\
\hline Eustala sp. 2 & 1 & & & & & 1 & & & 1 & 2 & 5 \\
\hline Eustala sp. 3 & & & 1 & & 1 & & & & & & 2 \\
\hline Eustala sp. 4 & & & & & & & 1 & & & & 1 \\
\hline Eustala sp. 5 & & & 1 & & & & & & & & 1 \\
\hline Eustala sp. 6 & & & & 1 & 1 & & & & & & 2 \\
\hline Eustala sp. 7 & 2 & & & & & 1 & & & & 1 & 4 \\
\hline Eustala sp. 8 & & & & & & & 1 & & & & 1 \\
\hline Hypognatha sp. 1 & 3 & 1 & 1 & & & & & & & & 5 \\
\hline Kapogea sexnotata & & & & & & & 1 & & & & 1 \\
\hline Mangora sp. 1 & 4 & 1 & 3 & 1 & 1 & 1 & 2 & & & & 13 \\
\hline Mangora sp. 2 & & & & 1 & 1 & & & 1 & & & 3 \\
\hline Mangora sp. 3 & 1 & & & & & & 1 & 5 & & & 7 \\
\hline Mangora sp. 4 & 3 & 1 & & & & & 1 & & & & 5 \\
\hline Mangora sp. 5 & & 1 & & 1 & & & & & & & 2 \\
\hline Mangora sp. 6 & 1 & & & & & & & 2 & & & 3 \\
\hline Metazygia sp. 1 & 1 & & 1 & & & & & & 1 & & 3 \\
\hline Metazygia sp. 2 & & & & & 1 & & 1 & & & & 2 \\
\hline Micratena aff. lata & & & & 1 & & & & & & & 1 \\
\hline Micratena clipeata & 1 & 1 & & & & & & 1 & & & 3 \\
\hline Micratena triangularispinosa & & & & & & 1 & & & & 3 & 4 \\
\hline Micratena pungens & & & & & 1 & & & & & 1 & 2 \\
\hline Ocrepeira sp. 1 & & & & & & & & 1 & 1 & & 2 \\
\hline Parawixia kochi & & 1 & & 1 & & 1 & & 1 & & & 4 \\
\hline Parawixia tarapoa & & & & & 1 & & & & & & 1 \\
\hline Spilasma duodecinguttata & 1 & & & & 1 & & & & & & 2 \\
\hline Wagneriana lechoza & & 1 & & & 1 & & & & & & 2 \\
\hline Wagneriana silvae & 1 & & & & & & & & 3 & & 4 \\
\hline Wagneriana undecimtuberculata & & & & & & & 2 & 1 & & & 3 \\
\hline Wagneriana sp. 1 & & & & 1 & & & 1 & & & & 2 \\
\hline \multicolumn{12}{|l|}{ Caponiidae } \\
\hline Nops sp. 1 & 1 & & & & & & & & & & 1 \\
\hline \multicolumn{12}{|l|}{ Clubionidae } \\
\hline Elaver sp. 1 & & & & 1 & & & & & & & 1 \\
\hline
\end{tabular}


Table 2 - Continuação

\begin{tabular}{|c|c|c|c|c|c|c|c|c|c|c|c|}
\hline Species/Areas & N 1 & N 2 & N 3 & N9 & $\mathrm{J} 19$ & $\mathrm{~J} 40$ & $\mathrm{~J} 46$ & $\mathrm{~J} 55$ & IGL & IGT & TOTAL \\
\hline \multicolumn{12}{|l|}{ Corinnidae } \\
\hline Abapeba taruma & & & & & & & 1 & 1 & & & 2 \\
\hline Castianeira sp. 1 & & & & & & 1 & & & & & 1 \\
\hline Castianeira sp. 2 & & & & & & & & 1 & 1 & & 2 \\
\hline Corinna aff. kochi sp. n. 1 & & & 1 & & & & & & & & 1 \\
\hline Corinna aff. recurva sp. n. 1 & 2 & 1 & & & & & & & 4 & & 7 \\
\hline Corinna aff. recurva sp. n. 2 & & & 1 & & & & 1 & & & & 2 \\
\hline Corinna aff. ducke sp. n. 1 & 1 & 1 & & & & & 1 & & & & 3 \\
\hline Corinna sp. 1 & & & 1 & & & & & & & 1 & 2 \\
\hline Corinna sp. 2 & & & 1 & & & & & & & & 1 \\
\hline Creugas sp. 1 & 1 & & & & & & & & & & 1 \\
\hline Falconina sp. 1 & & & & & & 1 & & & & & 1 \\
\hline Methesis sp. 1 & & & & & & 1 & & & & & 1 \\
\hline Myrmecium sp. 1 & 3 & 1 & 1 & 2 & 2 & 1 & & 2 & 4 & & 16 \\
\hline Myrmecium sp. 2 & 1 & & & & & & & & & & 1 \\
\hline Myrmecium sp. 3 & & & & & 1 & & & & & & 1 \\
\hline Parachemmis sp. n. 1 & 1 & & & & & & & & & & 1 \\
\hline Simonestus sp. n. 1 & & 2 & & & & & & & & 2 & 4 \\
\hline Sphecotypus sp. 1 & & & & & & & 1 & & & & 1 \\
\hline Stethorrhagus lupulus & 1 & & & & & 1 & & & & & 2 \\
\hline Trachelas sp. 1 & & & & & & & & 1 & 1 & & 2 \\
\hline Trachelas sp. 2 & & & 3 & & 1 & & & & & & 4 \\
\hline Trachelas sp. 3 & & & & & & & & & 1 & & 1 \\
\hline Trachelas sp. 4 & & & & 1 & & & & & & & 1 \\
\hline
\end{tabular}

Ctenidae

\begin{tabular}{|c|c|c|c|c|c|c|c|c|c|c|c|}
\hline Ancylometes amazonicus & & & & 1 & & & & & & & 1 \\
\hline Ancylometes rufus & 4 & 2 & 1 & 3 & & 1 & 2 & 3 & 5 & 3 & 24 \\
\hline Ancylometes sp. 1 & 1 & & & & & & & & & & 1 \\
\hline Centroctenus auberti & & & & & & 1 & & & & & 1 \\
\hline Centroctenus sp. 1 & 2 & & 2 & 3 & & & & 1 & 1 & 1 & 10 \\
\hline Centroctenus sp. 2 & & 5 & & & & & 1 & 1 & 1 & & 8 \\
\hline Centroctenus sp. 3 & & & & 2 & & & & & & & 2 \\
\hline Ctenus amphora & 2 & & & 6 & & & & & & & 8 \\
\hline Ctenus crulsi & 4 & 2 & 6 & 3 & & 4 & 2 & 4 & 2 & 1 & 28 \\
\hline Ctenus inaja & 1 & & & & & & & & & 1 & 2 \\
\hline Ctnenus minor & & & & 2 & & & & & & & 2 \\
\hline Ctenus sp. 1 & & & 1 & & & 1 & 2 & & & & 4 \\
\hline Ctenus sp. 2 & 2 & 2 & 2 & 1 & & 1 & 2 & 5 & 7 & 4 & 26 \\
\hline Ctenus sp. 3 & 1 & 1 & 1 & & 1 & & 1 & & 2 & & 7 \\
\hline Ctenus sp. 4 & & & & & & & & & 1 & & 1 \\
\hline Ctenus sp. 5 & & & & & & 1 & & & & & 1 \\
\hline Ctenus aff. villasboasi sp.1 & & & & & & 1 & & 1 & & & 2 \\
\hline Ctenus villasboasi & & 1 & 1 & & & 1 & & & & & 3 \\
\hline Cupiennius celerrimus & 2 & 1 & 3 & & 4 & 1 & 3 & 1 & 4 & 7 & 26 \\
\hline Enoploctenus mapia & & 1 & 2 & 1 & & & & & & & 4 \\
\hline Gephyroctenus sp. 1 & & & & & & & & & & 1 & 1 \\
\hline Gephyroctenus sp. 2 & & 2 & & & & & & & 4 & 1 & 7 \\
\hline
\end{tabular}


ACTA

AMAZONICA

A structured inventory of spiders (Arachnida, Araneae) in natural and artificial forest gaps at Porto Urucu, Western Brazilian Amazonia

Table 2 - Continuação

\begin{tabular}{|c|c|c|c|c|c|c|c|c|c|c|c|}
\hline Species/Areas & N 1 & N 2 & N 3 & N9 & $\mathrm{J} 19$ & $\mathrm{~J} 40$ & $\mathrm{~J} 46$ & $\mathrm{~J} 55$ & IGL & IGT & TOTAL \\
\hline Gephyroctenus sp. 3 & & & 1 & & & & & & & & 1 \\
\hline Phoneutria fera & & & 1 & 1 & & & 1 & & & & 3 \\
\hline Phoneutria reidyi & & & & & & & & 1 & 2 & & 3 \\
\hline \multicolumn{12}{|l|}{ Deinopidae } \\
\hline Deinops sp. 1 & & 1 & & 1 & & 2 & & & 1 & & 5 \\
\hline \multicolumn{12}{|l|}{ Dictynidae } \\
\hline Dictynidae sp. 1 & & & & & & & & & 1 & & 1 \\
\hline \multicolumn{12}{|l|}{ Dipluridae } \\
\hline Diplura sp. 1 & 1 & & & & & & & & & & 1 \\
\hline \multicolumn{12}{|l|}{ Hahniidae } \\
\hline Gen. sp. 1 & & & & & & & & 1 & & & 1 \\
\hline \multicolumn{12}{|l|}{ Hersiliidae } \\
\hline Ypypuera crucifera & 1 & & & & & & & & & 1 & 2 \\
\hline \multicolumn{12}{|l|}{ Linyphiidae } \\
\hline Gen. sp. 1 & & 1 & & & 5 & & & & & & 6 \\
\hline Gen. sp. 2 & 1 & & & & & & & 1 & & & 2 \\
\hline Gen. sp. 3 & & & & & & & & 1 & & & 1 \\
\hline Gen. sp. 4 & & & & & & & 1 & & & & 1 \\
\hline Gen. sp. 5 & 1 & & & & & & & & & & 1 \\
\hline Gen. sp. 6 & & 1 & & & & & & & & & 1 \\
\hline Dubiaranea sp. 1 & 5 & & 2 & & 12 & 12 & & 3 & 1 & & 35 \\
\hline Dubiaranea sp. 2 & 1 & & & & & & 2 & & & & 3 \\
\hline Dubiaranea sp. 3 & 1 & & & & & & & & & & 1 \\
\hline Meioneta sp. 1 & 1 & & & & & & & & & & 1 \\
\hline Sphecozone sp. 1 & & & & & 1 & & & & & & 1 \\
\hline Sphecozone sp. 2 & 3 & & & & 6 & & 5 & 2 & & & 16 \\
\hline Sphecozone sp. 3 & & & & & & & 1 & & & & 1 \\
\hline Sphecozone sp. 4 & 2 & & & & & & 1 & 1 & & & 4 \\
\hline Sphecozone sp. 5 & & & & & & & & 1 & & & 1 \\
\hline Sphecozone sp. 6 & & & & & & & & 1 & & & 1 \\
\hline \multicolumn{12}{|l|}{ Lycosidae } \\
\hline Aglaoctenus castaneus & & 1 & & & & & & & & & 1 \\
\hline Gen. sp. 1 & & & & & 7 & 17 & 27 & 64 & 8 & & 123 \\
\hline Gen. sp. 2 & & & & & 6 & 1 & 2 & 1 & & & 10 \\
\hline Gen. sp. 3 & & & & & & & 1 & & & & 1 \\
\hline \multicolumn{12}{|l|}{ Mimetidae } \\
\hline Ero sp. 1 & 1 & 1 & & 2 & & & 1 & & & & 5 \\
\hline Ero sp. 2 & & & & & & & & 1 & & & 1 \\
\hline Ero sp. 3 & & & & & & & & 1 & & & 1 \\
\hline Ero sp. 4 & & & & & & & & 2 & & & 2 \\
\hline Mimetus sp. 1 & & 1 & & & & & & & & & 1 \\
\hline Mimetus sp. 2 & & & & 1 & & & & & & & 1 \\
\hline Mimetus sp. 3 & & 1 & & & & & & & 1 & & 2 \\
\hline \multicolumn{12}{|l|}{ Mysmenidae } \\
\hline Gen. sp. 1 & 2 & 3 & 10 & & & & 1 & & & & 16 \\
\hline Gen. sp. 2 & & 4 & & & & & & & & & 4 \\
\hline
\end{tabular}


Table 2 - Continuação

\begin{tabular}{|c|c|c|c|c|c|c|c|c|c|c|c|}
\hline Species/Areas & N 1 & N 2 & N 3 & N9 & $\mathrm{J} 19$ & $\mathrm{~J} 40$ & $\mathrm{~J} 46$ & $\mathrm{~J} 55$ & IGL & IGT & TOTAL \\
\hline \multicolumn{12}{|l|}{ Miturgidae } \\
\hline Teminius insularis & & & & & 3 & 1 & & & & & 4 \\
\hline \multicolumn{12}{|l|}{ Nephilidae } \\
\hline Nephila clavipes & & & & & & & & & & 1 & 1 \\
\hline \multicolumn{12}{|l|}{ Ochyroceratidae } \\
\hline Gen. sp. 1 & & & & & & 1 & 1 & & & & 2 \\
\hline Gen. sp. 2 & 4 & 2 & 3 & 5 & & 2 & 3 & 1 & & & 20 \\
\hline Gen. sp. 3 & 6 & 7 & 17 & 8 & 2 & 4 & 4 & 1 & & & 49 \\
\hline Gen. sp. 4 & & & & & 3 & & & 1 & & & 4 \\
\hline Gen. sp. 5 & & & 2 & 1 & 1 & 4 & & & & & 8 \\
\hline \multicolumn{12}{|l|}{ Oonopidae } \\
\hline Coxapopha sp. 1 & & & 1 & & & & & & & & 1 \\
\hline Dysderina sp. 1 & & 1 & 1 & & 3 & 2 & & 5 & & & 12 \\
\hline Gamasomorphinae sp. 1 & & & & & 2 & 1 & 2 & 1 & & & 6 \\
\hline Gamasomorphinae sp. 2 & 1 & & & & & 1 & & & & & 2 \\
\hline Gamasomorphinae sp. 3 & & & & 2 & & & & & & & 2 \\
\hline Gamasomorphinae sp. 4 & & & 1 & & & & & & & & 1 \\
\hline Neoxyphinus sp. 1 & 4 & 5 & 2 & 1 & 1 & 1 & & 2 & & & 16 \\
\hline Neoxyphinus sp. 2 & & 1 & 2 & & 5 & 1 & 1 & 4 & 1 & & 15 \\
\hline Neoxyphinus sp. 3 & 1 & 3 & 3 & 3 & 1 & 2 & 1 & 3 & & & 17 \\
\hline Neoxyphinus sp. 4 & & & & & & 1 & & & & & 1 \\
\hline Oonopinae sp. 1 & & 1 & 1 & & & 1 & 1 & 3 & & & 7 \\
\hline Oonopinae sp. 2 & & 2 & & 1 & & & 1 & & & & 4 \\
\hline Oonopinae sp. 3 & & & & & & 3 & & & & & 3 \\
\hline Oonops sp. 1 & & 2 & & 2 & & & & & & & 4 \\
\hline Oonops sp. 2 & & & & & & & 1 & & & & 1 \\
\hline Scaphiella sp. 1 & 1 & 2 & & & & 1 & & & & & 4 \\
\hline
\end{tabular}

Oxyopidae

Oxyopes sp. 1

Oxyopes sp. 2

Oxyopes sp. 3

Tapinillus longipes

Tapponia sp. 1

Tapponia sp. 2

Palpimanidae

Otiothops sp. 1

Pholcidae

Gen. sp. 1

Gen. sp. 2

Gen. sp. 3

Gen. sp. 4

Gen. sp. 5

Carapoia ocaina

Mesabolivar sp. 1

Mesabolivar aurantiacus

Metagonia samiria

Metagonia sp. 1

\begin{tabular}{|c|c|c|c|c|c|c|}
\hline & 1 & & & & & 1 \\
\hline & 3 & & & & & 3 \\
\hline \multirow[t]{2}{*}{15} & & & & & & 15 \\
\hline & & 1 & & & & 1 \\
\hline \multirow[t]{13}{*}{1} & & 1 & & 1 & 1 & 6 \\
\hline & & & & 3 & & 4 \\
\hline & & 1 & & & & 1 \\
\hline & & & & 1 & & 2 \\
\hline & & & & & 2 & 2 \\
\hline & & & & & 3 & 3 \\
\hline & & & & & & 2 \\
\hline & & & & 1 & & 1 \\
\hline & 1 & 1 & & 1 & 1 & 12 \\
\hline & & 1 & & & 1 & 3 \\
\hline & & & 1 & 2 & 2 & 9 \\
\hline & & & & 1 & 1 & 2 \\
\hline & & & & 1 & 2 & 3 \\
\hline
\end{tabular}


ACTA

AMAZONICA

A structured inventory of spiders (Arachnida, Araneae) in natural and

artificial forest gaps at Porto Urucu, Western Brazilian Amazonia

Table 2 - Continuação

\begin{tabular}{|c|c|c|c|c|c|c|c|c|c|c|c|}
\hline Species/Areas & N1 & $\mathrm{N} 2$ & N 3 & N 9 & $\mathrm{~J} 19$ & $\mathrm{~J} 40$ & $\mathrm{~J} 46$ & J 55 & IGL & IGT & TOTAL \\
\hline Pisaboa aff. silvae & & & & & & & & & & 1 & 1 \\
\hline \multicolumn{12}{|l|}{ Pisauridae } \\
\hline Architis sp. 1 & & 1 & & 2 & 1 & & 2 & 1 & 2 & & 9 \\
\hline Gen. sp. 1 & & & & & & 4 & & & & & 5 \\
\hline Gen. sp. 2 & & & & & & & & & & 2 & 2 \\
\hline Thaumasia sp. 1 & & & & & & & 2 & 10 & & & 12 \\
\hline Thaumasia sp. 2 & & & 1 & & & & & 7 & 1 & 2 & 11 \\
\hline Thaumasia sp. 3 & & 1 & 1 & & & & & & 1 & 1 & 4 \\
\hline \multicolumn{12}{|l|}{ Salticidae } \\
\hline Gen. sp. 1 & & & & & 2 & & 4 & 5 & & & 11 \\
\hline Gen. sp. 2 & 4 & 1 & & & 1 & & 3 & 1 & & 4 & 14 \\
\hline Gen. sp. 3 & & & & 1 & & & & & & & 1 \\
\hline Gen. sp. 4 & 2 & 2 & & & & & & & 1 & 4 & 9 \\
\hline Gen. sp. 5 & & 1 & & & & & & & & & 1 \\
\hline Gen. sp. 6 & 1 & & & 1 & 1 & & & & & & 3 \\
\hline Gen. sp. 7 & & & & & 1 & & & & & & 1 \\
\hline Gen. sp. 8 & 1 & & & & & & & & & & 1 \\
\hline Gen. sp. 9 & & & & & & 1 & & & & & 1 \\
\hline Gen. sp. 10 & & 1 & & & & & & 1 & & & 2 \\
\hline Gen. sp. 11 & 1 & & & & & & & & & & 1 \\
\hline Gen. sp. 12 & & & 1 & 1 & & & & & & & 2 \\
\hline Gen. sp. 13 & & & & & 1 & & & & 1 & & 2 \\
\hline Gen. sp. 14 & & & & & & & & & & 1 & 1 \\
\hline Gen. sp. 15 & 1 & & & & & 1 & 1 & & 2 & 1 & 6 \\
\hline Gen. sp. 17 & & & & 1 & & 1 & & & 1 & & 3 \\
\hline Gen. sp. 18 & & & 1 & & & & & & & & 1 \\
\hline Gen. sp. 19 & & & & & 1 & & & & & & 1 \\
\hline Gen. sp. 20 & & & & & & 1 & 1 & 2 & & & 4 \\
\hline Gen. sp. 21 & & & & & & & & & & 2 & 2 \\
\hline Gen. sp. 22 & & & 1 & & & & & & & & 1 \\
\hline Gen. sp. 23 & & & 1 & & & & & & 1 & & 2 \\
\hline Gen. sp. 24 & 1 & & 1 & & & & & & & & 2 \\
\hline Gen. sp. 25 & 1 & & & & & & & & 1 & & 2 \\
\hline Gen. sp. 26 & & & & & 2 & & 1 & & & & 3 \\
\hline Gen. sp. 27 & 1 & & & & & & & & & 1 & 2 \\
\hline Gen. sp. 28 & & 1 & & & & & & & & & 1 \\
\hline Gen. sp. 29 & & & & & & & & & 2 & & 2 \\
\hline Gen. sp. 30 & & & & & & & & & & 2 & 2 \\
\hline Gen. sp. 31 & & 1 & & & & & & & & 1 & 2 \\
\hline Gen. sp. 32 & & & & 1 & & & & & & & 1 \\
\hline Gen. sp. 33 & & & & & & & 1 & & & & 1 \\
\hline Gen. sp. 34 & & 1 & & & & & 1 & & & & 2 \\
\hline Gen. sp. 35 & & 1 & & 1 & & & & & & & 2 \\
\hline Gen. sp. 36 & & & & & & & 2 & & & & 2 \\
\hline Gen. sp. 37 & 1 & & 2 & 2 & & & & & & & 5 \\
\hline Gen. sp. 38 & 1 & & 1 & & & & & 1 & & & 3 \\
\hline Gen. sp. 39 & & 3 & & 2 & 1 & 1 & & 1 & & & 8 \\
\hline
\end{tabular}


Table 2 - Continuação

\begin{tabular}{|c|c|c|c|c|c|c|c|c|c|c|c|}
\hline Species/Areas & N1 & N 2 & N 3 & N 9 & $\mathrm{~J} 19$ & $\mathrm{~J} 40$ & $\mathrm{~J} 46$ & J 55 & IGL & IGT & TOTAL \\
\hline Gen. sp. 40 & & & & & 1 & & & & & & 1 \\
\hline Gen. sp. 41 & & & & & 1 & & & & & & 1 \\
\hline Gen. sp. 42 & & & & & & 1 & & & & & 1 \\
\hline Gen. sp. 43 & & & & 1 & & & & & & & 1 \\
\hline Gen. sp. 44 & 1 & 1 & & & & 1 & & & & & 3 \\
\hline Gen. sp. 45 & & & & & 1 & & & 1 & & & 2 \\
\hline Gen. sp. 46 & & & & 1 & & & & & & & 1 \\
\hline Gen. sp. 47 & & & & 1 & & & & & & & 1 \\
\hline Gen. sp. 48 & & & & 1 & & & & & & & 1 \\
\hline Gen. sp. 49 & & & & & & 2 & & & & & 2 \\
\hline Gen. sp. 50 & & 1 & & & & & & & & & 1 \\
\hline Gen. sp. 52 & & & & 1 & 1 & & & & & & 2 \\
\hline Gen. sp. 53 & & & & 2 & & & & & & & 2 \\
\hline Chira sp. 1 & 1 & 2 & & & & 1 & & & & 2 & 6 \\
\hline Chira sp. 2 & 1 & 1 & & & & & 1 & 1 & & & 4 \\
\hline Chira sp. 3 & 1 & & & & 1 & & 1 & & & & 3 \\
\hline Chira sp. 4 & & & 1 & & & & & & & 1 & 2 \\
\hline Chira sp. 5 & & 1 & 1 & & & 2 & & 1 & 2 & 2 & 9 \\
\hline Cotinusa sp. 1 & & & & & & & 1 & & & & 1 \\
\hline Lyssomanes sp. 1 & 1 & 1 & & & & 1 & & 1 & 1 & 1 & 6 \\
\hline Phiale sp. 1 & & & & & & & 1 & & & & 1 \\
\hline Phiale sp. 2 & & & & & & 1 & & & & & 1 \\
\hline \multicolumn{12}{|l|}{ Scytodidae } \\
\hline Scytodes sp. 1 & & 2 & & & & & & & & & 2 \\
\hline Scytodes auricula & 1 & 1 & 2 & 1 & & 1 & 1 & 1 & 2 & 3 & 13 \\
\hline Scytodes romitti & 1 & 1 & & & & & & & & & 2 \\
\hline \multicolumn{12}{|l|}{ Senoculidae } \\
\hline Senocolus sp. 1 & & & & & & & & 1 & & & 1 \\
\hline \multicolumn{12}{|l|}{ Sparassidae } \\
\hline Gen. sp. 1 & & & 1 & & & & & & & & 1 \\
\hline Gen. sp. 3 & 1 & & & & & & & & & & 1 \\
\hline Gen. sp. 4 & & & & & & & 1 & & & 1 & 2 \\
\hline Gen. sp. 5 & & & & & & & 2 & & & & 2 \\
\hline Gen. sp. 6 & & 1 & & & & 1 & & & 1 & & 3 \\
\hline Sparianthinae sp. 1 & & & & & & & 1 & & & & 1 \\
\hline Pseudosparianthis sp. 1 & 1 & & & & 1 & & & 1 & & & 3 \\
\hline \multicolumn{12}{|l|}{ Symphytognathidae } \\
\hline Gen. sp. 1 & 1 & 2 & 2 & & & & & 1 & & & 6 \\
\hline Gen. sp. 2 & & 1 & & & & & & & & & 1 \\
\hline Gen. sp. 3 & & & & & 1 & & & & & & 1 \\
\hline \multicolumn{12}{|l|}{ Synotaxidae } \\
\hline Gen. sp. 1 & & 1 & & & & 1 & & & & & 2 \\
\hline Gen. sp. 2 & & & & 1 & & & & & 1 & & 2 \\
\hline Gen. sp. 3 & & & & & & & & 1 & & & 1 \\
\hline \multicolumn{12}{|l|}{ Tetragnathidae } \\
\hline Gen. sp. 1 & & & & & & 1 & & & & & 1 \\
\hline Gen. sp. 2 & 1 & & & 2 & 2 & & 5 & & & & 10 \\
\hline
\end{tabular}


ACTA

AMAZONICA

A structured inventory of spiders (Arachnida, Araneae) in natural and

artificial forest gaps at Porto Urucu, Western Brazilian Amazonia

Table 2 - Continuação

\begin{tabular}{|c|c|c|c|c|c|c|c|c|c|c|c|}
\hline Species/Areas & N1 & $\mathrm{N} 2$ & N 3 & N 9 & $\mathrm{~J} 19$ & $\mathrm{~J} 40$ & J 46 & J 55 & IGL & IGT & TOTAL \\
\hline Chrysometa sp. 1 & & 1 & & & & & & & 1 & 1 & 3 \\
\hline Leucauge sp. 1 & 3 & & & & & & & 1 & & & 4 \\
\hline Leucauge sp. 2 & 1 & & 1 & & & & & & & & 2 \\
\hline Leucauge sp. 3 & 1 & & & & & & & & & & 1 \\
\hline Leucauge sp. 4 & & & & & 1 & & & & & & 1 \\
\hline Leucauge sp. 5 & & & & & 1 & & & 1 & & & 2 \\
\hline Leucauge sp. 6 & & & & & 1 & & & & & & 1 \\
\hline Leucauge sp. 8 & & 1 & & & & & & & & & 1 \\
\hline Leucauge sp. 9 & & & & & & & 1 & 3 & & & 4 \\
\hline Leucauge sp. 10 & & & & & & & & & 1 & & 1 \\
\hline Leucauge sp. 11 & & & & & 1 & & & & & & 1 \\
\hline Tetragnatha sp. 1 & & & & & & & & 1 & & & 1 \\
\hline \multicolumn{12}{|l|}{ Theridiidae } \\
\hline Gen. sp. 1 & & 1 & & & & & & & 1 & & 2 \\
\hline Gen. sp. 2 & & & & & & & & & 2 & & 2 \\
\hline Gen. sp. 3 & & & & & & & & & 2 & & 2 \\
\hline Gen. sp. 4 & & & & & & & & 1 & & & 1 \\
\hline Gen. sp. 5 & & & & & & & & & & 1 & 1 \\
\hline Gen. sp. 6 & & & & & & & & 1 & & & 1 \\
\hline Gen. sp. 7 & & & 2 & & & & & & 1 & & 3 \\
\hline Gen. sp. 8 & & 1 & & & & & & & & & 1 \\
\hline Gen. sp. 9 & & & & & & & 1 & & & & 1 \\
\hline Gen. sp. 10 & & 1 & & & & & & 1 & & & \\
\hline Gen. sp. 11 & & 1 & & & & & & & & & 1 \\
\hline Gen. sp. 12 & & & & & & & & & 1 & & 1 \\
\hline Gen. sp. 13 & 1 & & & & & & & & & & 1 \\
\hline Gen. sp. 14 & & & & & & & & 1 & & & 1 \\
\hline Gen. sp. 15 & & & & 1 & & & & & & & 1 \\
\hline Gen. sp. 16 & & & & 1 & & & & & & & 1 \\
\hline Gen. sp. 17 & 1 & & & & & & & & & 1 & 2 \\
\hline Gen. sp. 18 & 1 & 1 & & & & & & & & 2 & 4 \\
\hline Gen. sp. 19 & & & & & & & & & & 1 & 1 \\
\hline Gen. sp. 20 & & 1 & & & & & & & & & 1 \\
\hline Gen. sp. 21 & & & & & & & & & & 1 & 1 \\
\hline Gen. sp. 22 & & & 1 & & & & & & & & 1 \\
\hline Gen. sp. 23 & & & & & & & & 1 & & & 1 \\
\hline Gen. sp. 24 & & 1 & & & & & & 1 & & & 2 \\
\hline Gen. sp. 25 & 1 & & & & & & & & & 2 & 3 \\
\hline Achaearanea sp. 1 & & & & & 1 & 1 & 1 & & & & 3 \\
\hline Achaearanea sp. 2 & 1 & & & & & 1 & & 1 & 2 & & 5 \\
\hline Achaearanea sp. 20 & & & & & & 1 & & & & & 1 \\
\hline Anelosimus sp. 1 & & & & & & & 40 & & & & 40 \\
\hline Argyrodes sp. 1 & 1 & & 1 & & 1 & & 1 & 1 & & & 5 \\
\hline Argyrodes sp. 2 & & & & & & & & & 1 & & 1 \\
\hline Argyrodes sp. 3 & & & & & & & 1 & & & & 1 \\
\hline Argyrodes sp. 5 & 3 & & & & & & & & & & 3 \\
\hline Argyrodes sp. 6 & & & & & & & & & 1 & & 1 \\
\hline
\end{tabular}


Table 2 - Continuação

\begin{tabular}{|c|c|c|c|c|c|c|c|c|c|c|c|}
\hline Species/Areas & N 1 & $\mathrm{~N} 2$ & N 3 & N 9 & $\mathrm{~J} 19$ & $\mathrm{~J} 40$ & J 46 & J 55 & IGL & IGT & TOTAL \\
\hline Argyrodes sp. 7 & & & 2 & & & & & & & & 2 \\
\hline Cerocida aff. strigosa & & & 1 & & & & & & & & 1 \\
\hline Chrosiotes sp. 1 & & & 1 & & & & & & & & 1 \\
\hline Chrosiotes sp. 2 & & & & & & & & & & 1 & 1 \\
\hline Chrosiotes sp. 3 & 1 & & & & & & & & & 1 & 2 \\
\hline Chrysso sp. 1 & 1 & 1 & & & & 1 & 1 & & & 1 & 5 \\
\hline Chrysso sp. 2 & & & & & & & & 1 & & & 1 \\
\hline Chrysso sp. 3 & & & & & & & & & & 1 & 1 \\
\hline Coleosoma acutiventer & & & & & 6 & & & & & & 6 \\
\hline Dipoena aff. atlantica & 2 & & & & & 1 & & & 1 & & 4 \\
\hline Dipoena aff. kuyuwini & & 1 & 1 & & & & & & & & 2 \\
\hline Dipoena aff. donaldi & & 1 & & 1 & & & 1 & & & & 3 \\
\hline Dipoena aff. tiro & & 2 & & & & & & & & & 2 \\
\hline Dipoena sp. 1 & 1 & & & 3 & 2 & 1 & & & 2 & & 9 \\
\hline Dipoena sp. 2 & 3 & & & & & 1 & & & & & 4 \\
\hline Dipoena sp. 3 & & & & & & & 1 & & & & 1 \\
\hline Dipoena sp. 4 & & & 1 & & & & & & & & 1 \\
\hline Dipoena sp. 5 & & & & & & & 1 & & & & 1 \\
\hline Dipoena sp. 6 & & 1 & & & & & & & & & 1 \\
\hline Dipoena sp. 7 & & & & 1 & & & & & & & 1 \\
\hline Dipoena sp. 8 & & & & & & & 1 & & & & 1 \\
\hline Dipoena sp. 9 & & & & & & & & & & 1 & 1 \\
\hline Echinotheridion sp. 1 & 1 & & & & & & & & & & 1 \\
\hline Episinus sp. 1 & 4 & 8 & 9 & 1 & 3 & 1 & 1 & 4 & 4 & 3 & 38 \\
\hline Episinus sp. 2 & 1 & 2 & 1 & 1 & & 2 & & 4 & & 1 & 12 \\
\hline Episinus sp. 3 & & 1 & & & & 1 & & & & 1 & 3 \\
\hline Episinus sp. 4 & & & & & & & & & & 1 & 1 \\
\hline Episinus sp. 5 & & & 1 & & 1 & & & & & & 2 \\
\hline Episinus sp. 6 & & & & 1 & & & & & & & 1 \\
\hline Episinus sp. 7 & & & & 1 & & & & & & & 1 \\
\hline Episinus sp. 8 & & & 1 & & & & 1 & & & 1 & 3 \\
\hline Stemmops sp. 1 & & & 1 & 2 & & 1 & & & & & 4 \\
\hline Stemmops sp. 2 & & & & & & & 1 & 1 & & & 2 \\
\hline Stemmops sp. 3 & 1 & & & & & & & & & & 1 \\
\hline Thwaitesia bracteata & 4 & 2 & & 4 & 1 & & & & & & 11 \\
\hline \multicolumn{12}{|l|}{ Theridiossomathidae } \\
\hline Gen. sp. 1 & & & & 1 & & & & & & & 1 \\
\hline Gen. sp. 2 & 1 & & & & & & & & & & 1 \\
\hline Gen. sp. 3 & & 1 & & & & & & & & & 1 \\
\hline Naatlo sp. 1 & & & & 1 & & 1 & & & & & 2 \\
\hline Naatlo sp. 2 & 2 & & & & & & & & & & 2 \\
\hline Naatlo sp. 3 & & & & 1 & & & 3 & & 1 & & 5 \\
\hline Theridiossoma sp. 1 & & & & 2 & & 1 & & & & & 3 \\
\hline Theridiossoma sp. 2 & & & & & & & & & 1 & & 1 \\
\hline Theridiossoma sp. 3 & & & & & & & 1 & & & & 1 \\
\hline \multicolumn{12}{|l|}{ Theraphosidae } \\
\hline Cyioscosmus sp. 1 & & & & 1 & & & & & & & 1 \\
\hline
\end{tabular}




\begin{tabular}{|c|c|c|c|c|c|c|c|c|c|c|c|}
\hline Species/Areas & N 1 & N 2 & N 3 & N 9 & $\mathrm{~J} 19$ & $\mathrm{~J} 40$ & $\mathrm{~J} 46$ & $\mathrm{~J} 55$ & IGL & IGT & TOTAL \\
\hline Theraphosinae sp. 1 & & & & & 1 & & & & & & 1 \\
\hline \multicolumn{12}{|l|}{ Thomisidae } \\
\hline Gen. sp. 1 & & 1 & & & & & & & & & 1 \\
\hline Ascentrocelus sp. 1 & 1 & & & & & & & & & & 1 \\
\hline Misumenops sp. 1 & & & & & & & & 1 & & & 1 \\
\hline Onocolus echinatus & & & & 1 & & & 1 & & 2 & & 4 \\
\hline Stephanopis sp. 1 & & 1 & 4 & & 2 & 1 & & 1 & & & 9 \\
\hline Stephanopis sp. 2 & & 1 & & & & & & & & & 1 \\
\hline Strophius sp. 1 & 1 & 1 & & & & & & & & & 2 \\
\hline Synaema sp. 1 & & & & & & 1 & & 1 & & & 2 \\
\hline Tmarus sp. 1 & & & & & & & & 1 & & & 1 \\
\hline Tmarus sp. 2 & & & & & & & 1 & & & & 1 \\
\hline Tmarus sp. 3 & 1 & & & & & & & & & & 1 \\
\hline Tmarus sp. 4 & & & & & & & 1 & & & & 1 \\
\hline Tmarus sp. 5 & & 2 & & & & & & & & & 2 \\
\hline Tmarus sp. 6 & & & 1 & & & & & & & & 1 \\
\hline Tmarus sp. 7 & & & & & & 1 & & & & & 1 \\
\hline Tmarus sp. 8 & & & & & & & & & & 1 & 1 \\
\hline Tmarus sp. 9 & & & & & & 1 & & & & & 1 \\
\hline \multicolumn{12}{|l|}{ Trechaleidae } \\
\hline Dossenus sp. 1 & & & & & & & & & 29 & 17 & 46 \\
\hline Dossenus sp. 2 & & & & & & & & & 7 & & 7 \\
\hline Dossenus sp. 3 & & & & & & & & 2 & 2 & 2 & 6 \\
\hline Trechalea sp. 1 & & 1 & & & & & & & & & 1 \\
\hline Trechalea sp. 2 & & & & & 1 & 1 & & & 3 & 1 & 6 \\
\hline Trechalea macconnelli & & & & & & & & & & 2 & 2 \\
\hline \multicolumn{12}{|l|}{ Uloboridae } \\
\hline Gen. sp. 1 & & & & & & & 1 & & & & 1 \\
\hline Miagrammopes sp. 1 & & & 1 & & & & & & & & 1 \\
\hline Philoponella sp. 1 & 1 & 1 & & & & & & & & & 2 \\
\hline Philoponella sp. 2 & 1 & 2 & & & & 1 & 1 & & & 1 & 6 \\
\hline Philoponella sp. 3 & & & & 1 & & & & & & & 1 \\
\hline Uloborus sp. 1 & & & 1 & & & & & 1 & & & 2 \\
\hline Zosis aff. geniculatus & & & 2 & & 1 & 1 & & & & & 4 \\
\hline Total & 168 & 159 & 153 & 116 & 158 & 140 & 206 & 222 & 163 & 127 & 1612 \\
\hline
\end{tabular}

\section{SPECIES RICHNESS AND SIMILARITY IN ARTIFICIAL AND NATURALS FOREST GAPS}

Excluding the occasional sampling, 3414 individuals were recorded in both artificial and natural forest gap areas. The 1321 adult specimens were identified as comprising 357 species or morphospecies recorded in 218 valid samples. To provide an indication of the total species richness present in the study site, the results of seven species richness estimators are made available for entire Porto Urucu dataset (Fig. 2). The estimates ranged from 662 species (Jackknife 2) to 435 species (Bootstrap). However, these results must be viewed cautiously because the inventory is not complete. Walther \& Moore (2005) stressed that each estimator has been developed to work best under the assumptions defined by a specific population model, which defines the population structure resulting from various community parameters. Thus, performance tests always must be carried out in order to verify which estimator fits best to the particular conditions of each inventory.

Toti et al. (2000), in testing a spider assemblage's database, indicated that a good way to test the performance of a given estimator is to inspect visually the shape of the curve, looking for trends toward the asymptote. The best estimators would be 


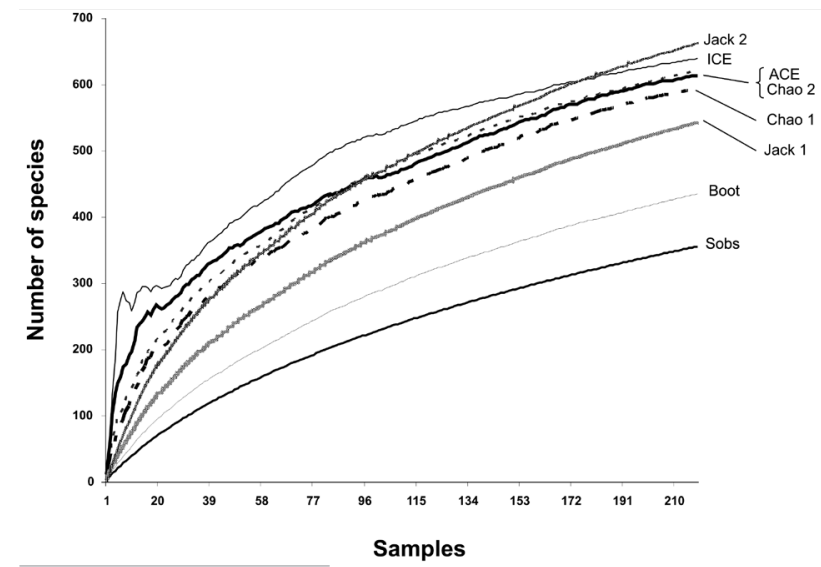

Figure 2 - Observed sampling accumulation curve and non parametric estimator performance for the data set of spiders collected in both artificial and natural forest gap areas in Porto Urucu, Coari, Amazonas, Brazil. The tested estimators are Jackknife 1 and 2, ICE, ACE, Chao 1 and 2, and the Bootstrap.

those which need less information to approach stability. In the case here presented, no estimator reached a clear asymptote; but after applying the above procedure, we verified that ICE was the best fit estimator to this data set, characterized by large numbers of singletons and uniques. ICE (incidencebased coverage estimator) is based on the proportion of species presented in the samples, relies on presence/absence data to quantify rarity and retrieves information from species represented by up to 10 individuals (Toti et al., 2000; Colwell, 2006). The preferred estimated number, produced by ICE, was 639 species. This is the second larger estimate, being surpassed only by Jackknife 2. However, it is not an absurd prediction because the only spider inventory from a nearby locality available to comparison, the one provided by Höfer $\&$ Brescovit (2001) for the RFAD, reported an observed (or minimal) species richness of 506 species.

Inspecting the individual-based rarefaction curves for both JAZ and NAT communities (Fig. 3) at the point that a comparison is possible ( $565^{\text {th }}$ individual added), JAZ accumulated 196 species, while NAT accumulated 228 species. These differences are significant given the calculated confidence interval $(\alpha=0.05)$. The rarefaction curve for NAT reached a higher species richness with fewer specimens than the JAZ curve, which needed to add 712 individuals to accumulate only 224 species at the end of data entry.

The values of the similarity indices for the JAZ and NAT communities were 0,34 and 0,48 for Kulczynski's and Sørensen's indexes, respectively. At the end of the observed sample-based accumulation curves, the samples related to natural forest gaps produced 239 species, while 230 species were recorded from the samples related to artificial forest gaps. Despite this apparently similar observed richness, only 112 species were common to these two treatments. Both

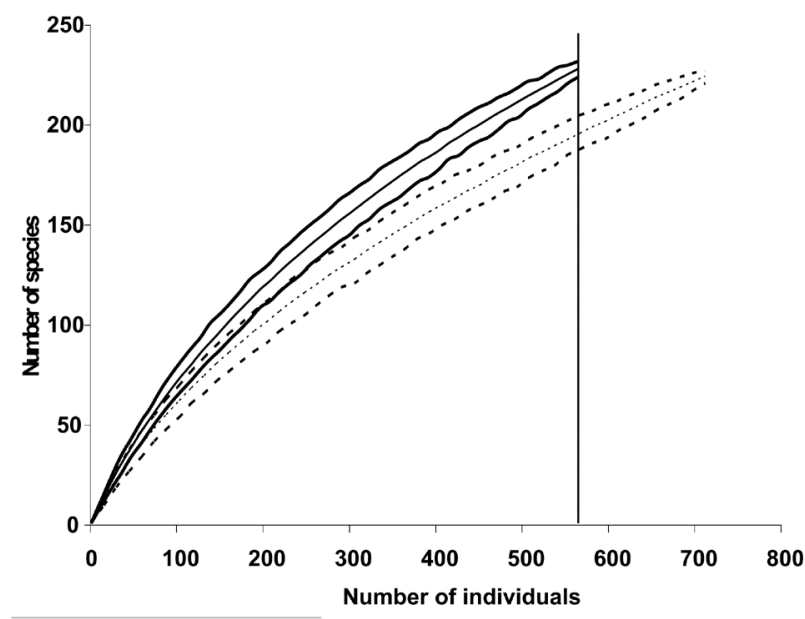

Figure 3 - Individual-based rarefaction curves for spiders collected in both artificial and natural forest gap areas in Porto Urucu, Coari, Amazonas, Brazil, with their respective $95 \%$ confidence interval lower and upper bounds. Solid lines, natural forest gap areas; dotted-lines, artificial forest gap areas.

indices above depicted considerable differences regarding spider species composition between these two communities. The differences between the two kinds of communities were detectable, even considering that the collecting effort was less intense in the center of both kinds of gaps than in its forested edges and adjacent forest matrix. In the natural forest gaps of Porto Urucu, the composition of the plant communities does not differ greatly from that of the original forest matrix, but the artificial forest gaps present more herbaceous and pioneers plants (Lima Filho et al. 2001). It is possible that the differences depicted in species richness and composition of species among the two spider communities reflect responses to differences in physiognomy and plant species composition of the gaps themselves, revealing a differential influence of the presence of each kind of gap in the forest matrix.

\section{LYCOSIDAE AND CTENIDAE AS ENVIRONMENTAL INDICATORS}

Comparing the families represented by more than 400 individuals (Fig. 4), a Mann Whitney test analyzing differences in abundance of Lycosidae in natural versus artificial forest gaps areas show that representatives of this family are much more abundant in JAZ areas than in NAT areas $(\mathrm{U}=0 ; \mathrm{p}=0.0209)$. On the other hand, $T$ test show that Ctenidae was more abundant in NAT than in JAZ areas $(t=3.289 ; \mathrm{p}=0.0166)$.

The great abundance of Ctenidae in NAT areas could be explained by a putative increase of suitable ground shelters for these spiders, provided in the center of the NAT gaps by the fallen trees. In fact, the gap centers contributed 202 individuals out of the 395 collected in NAT areas (51\%). However, the results of the $\mathrm{T}$ test could be biased by the rarity of Ctenidae in the center of the JAZ gaps (only 31 out of all 179 individuals in JAZ, or 17\%). 


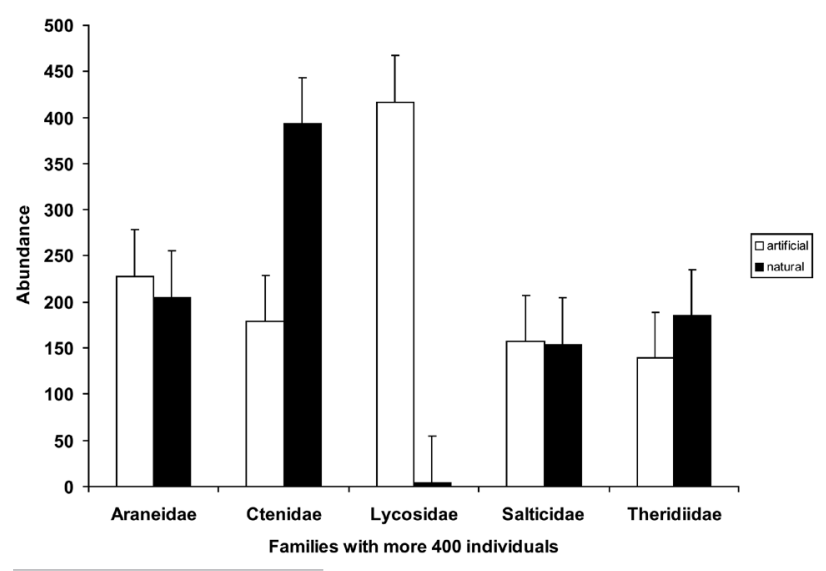

Figure 4 - Distribution of the most common spider families ( $\geq 400$ individuals), with their respective standard errors, between natural and artificial forest gap areas in Porto Urucu, Coari, Amazonas, Brazil.

The large number of representatives of the family Lycosidae $(\mathrm{N}=432)$ is an unusual result for tropical rain forest sites. From all individuals collected in Porto Urucu, 417 were from JAZ communities and only 15 were from NAT areas or from the surroundings of the creeks. Moreover, except for eight specimens from the adjacent forest matrix, all specimens of Lycosidae obtained in JAZ areas were collected in the gaps themselves. Jocqué \& Alderweireldt (2005) stated that lycosids are consistently more abundant in areas covered by herbaceous layer, which is negatively correlated with the degree of canopy closure. In Porto Urucu, the natural forest gaps are surrounded by the primary forest and the canopy discontinuity is small. On the other hand, the artificial forest gaps represent a large canopy discontinuity, where in some areas, only herbaceous plants are present.

The explosive abundance of Lycosidae in artificial gaps, combined with its extreme rarity in the wider forest matrix qualifies it as an excellent environmental indicator of forest degraded areas. The incidence and relative abundance of Ctenidae could be also monitored, providing a complementary indicator of recovering gap areas.

\section{RESEARCH PERSPECTIVES IN PORTO URUCU}

The continuity of the project "Dinâmica de clareiras sob impacto da exploração petrolífera" (DICLA), which aims to address some fundamental questions involving community dynamics in artificial gaps in Porto Urucu, allowed us to considerably increase the sampling effort in the study area. Since 2005, further studies on the spider fauna were carried out as part of the second author's PhD project. This project applied a sampling protocol designed to maximize the acquiring of data related to spider fauna dynamics in artificial forest gaps with various degrees of regeneration, grouping gaps in three blocks, each one with high sample representation levels. Several occasional and fogging samples, as well as nearly 800 samples from a protocol composed by beating tray, nocturnal hand search and Winkler extractors, are available from this study. Adding information from these samples to the present dataset may help to better quantify the environmental impact of the artificial gaps in the spider communities present in the forest matrix of Porto Urucu.

\section{ACKNOWLEDGMENTS}

Logistic support in the field was provided by Petrobras S.A.; we are grateful to M.B. Martins (MPEG) for suggestions regarding the sampling design, to D.D. Guimarães and J.O. Dias (MPEG) for helping during field work, to A. D. Brescovit (Instituto Butantan, São Paulo, IBSP) for information on previous records of spider species in Porto Urucu and to L. S. Carvalho (MPEG UFPI), A. J. Santos (Universidade Federal de Minas Gerais) and T. Gardner (University of East Anglia Cambridge, UK) for useful comments to the manuscript; A.D. Brescovit, C.A. Rheims (IBSP), N. Abrahim, J.A.P. Barreiros, D.R. Santos-Souza (MPEG), E.H. Buckup and M.A. Marques (Museu de Ciências Naturais, Porto Alegre) kindly helped to identify the material; the authors were supported by CNPq (SCD DR grant \#140722/2006-8; ABB PQ grant \#303591/2006-3).

\section{LITERATURE CITED}

Ayres, M.; Ayres Jr., M.; Ayres, D.L.; Santos, A.S. 2005. BioEstat 4.0: statistical applications in biological sciences and medical areas. Sociedade Civil Mamirauá. (in portuguese)

Borges, S.H.; Brescovit, A.D. 1996. Preliminary inventory of arachnids (Araneae) in two localities of Occidental Amazonia. Boletim do Museu Paraense Emílio Goeldi, Série Zoologia, 12(1): 9-21. (in portuguese)

Bonaldo, A.B.; Marques, M.A.L.; Pinto-da-Rocha, R. \& Gardner, T. 2007. Species richness and community structure of arboreal spider assemblages in fragments of three forest types at Banhado Grande wet plain, Gravataí River, Rio Grande do Sul, Brazil. Iheringia, Série Zoologia, 97(2): 143-151.

Brescovit, A. D. 1996. Revision of the genus Centroctenus MelloLeitão (Araneae, Ctenidae, Cteninae). Revista Brasileira de Entomologia, 40: 301-313. (in portuguese)

Brescovit, A.D.; Bonaldo, A.B.; Bertani, R.; Rheims, C.A. 2002 Araneae. In: Adis, J. (ed). Amazonian Arachida and Myriapoda. Pensoft: 303-343.

Brescovit, A,D.; Bertani, R; Pinto-da-Rocha, R.; Rheims, C.A. 2004. Arachnids of Juréia-Itatins Ecology Station: preliminary inventory and natural history. In: Marques, O.A.V.; Duleba, W. (eds). Juréia-Itatins Ecology, flora and fauna. Holos, Ribeirão Preto: 198-221. (in portuguese)

Brescovit, A. D.; Oliveira, M. E. do E. S. 1994. Rhoicinus urucu, a new species of Rhoicininae from Amazonian region (Araneae, Trechaleidae). Biociências, 2: 63-69. (in portuguese) 
Brescovit, A. D.; von Eickstedt, V. R. D. 1995. Ocurrence of Cupiennius Simon in South America and redescription of Cupiennius celerrimus Simon (Araneae, Ctenidae). Revista Brasileira de Zoologia, 12: 641-646. (in portuguese)

Coddington, J.A.; Griswold, C.E.; Dávila, D.S.; Peñaranda, E.; Larcher, S.F. 1991. Designing and testing sampling protocols to estimate biodiversity in tropical ecosystems. In: Dudley, E.C. (ed). Unity of Evolutionary Biology: Proceedings of The Fourth International Congress of Systematic and Evolutionary Biology. Dioscorides Press: 44-60.

Coddington, J.A.; Young, L.H.; Coyle, F.A. 1996. Estimating spider species richness in a southern Appalachian cove hardwood forest. Journal of Arachnology, 24: 111-128.

Colwell, R.; Coddington, J.A. 1994. Estimating terrestrial biodiversity through extrapolation. Philosophical Transactions of the Royal Society of London B, 345: 101-118.

Colwell, R.K. 2006. Estimates: Estimation of species richness and shared species from samples. Version 7.5. http://viceroy.eeb.uconn. edu/EstimateS.

Gotelli, N.J.; Entsminger, G.L. 2001. EcoSim: Null models software for ecology. Version 7.0. Acquired Intelligence Inc. \& Kesey-Bear. http://homepages.together.net/ - gentsmin/ecosim.htm.

Höfer, H.; Brescovit, A. D. 2000. A revision of the Neotropical spider genus Ancylometes Bertkau (Araneae: Pisauridae). Insect Systematic and Evolution, 31: 323-360.

Höfer, H.; Brescovit, A.D. 2001. Species and guild structure of a Neotropical spider assemblage (Araneae) from Reserva Ducke, Amazonas, Brazil. Andrias, 15: 99-119.

Höfer, H., Brescovit, A. D.; Gasnier, T. 1994. The wandering spiders of the genus Ctenus (Ctenidae, Araneae) of Reserva Ducke, a rainforest reserve in central Amazonia. Andrias, 13: 81-98.

Jocqué, R.; Alderweireldt, M. 2005. Lycosidae: the grassland spiders. Acta zoologica bulgarica, suppl. 1: 125-130.

Krebs, C.J. 1999. Ecological methodology. Addison Wesley Longman. $620 \mathrm{p}$.

Lima Filho, D.A.; Matos, F.D.A.; Amaral, I.L.; Revilla, J.; Coêlho, L.S.; Ramos, J.F.; Santos, J.L. 2001. Floristic inventory in a terra frme ombrofilous forest at Urucu River - Amazonas, Brasil. Acta Amazonica 31: 565-579. (in portuguese)
RADAM-BRASIL. 1978. National Integration Project. Natural Resources Survey. Vol.18 (Manaus - RADAM/Projeto - DBPM). Ministério das Minas e Energia. 626 p. (in portuguese)

Ribeiro, J.E.L. da S.; Hopkins, M.J.G.; Vicentini, A.; Sothers, C.A.; Costa, M.A. da S.; Brito, J.M. de.; Sousa, M.A. de.; Martins, L.H.P.; Lohmann, L.G.; Assunção, P.A.C.L.; Pereira, E. da C.; Silva. C.F. da.; Mesquita, M.R.; Procópio, L.C. 1999. Reserva Ducke Flora: Guide of identification of vascular plants in a 'terra firme' forest at Central Amazonia. Instituto Nacional de Pesquisas da Amazônia, Manaus, Amazonas. 799 pp. (in portuguese)

Silva, D. 1992. Observations on the diversity and distribution of the spiders of Peruvian montane forests. Memorias Del Museo de Historia Natural, U.N.S.M. (Lima), 21:31-37.

Silva, D. 1996. Species composition and community structure of Peruvian Rainforest spiders: a case study from a seasonally innunded forest along the Samiria River. Revue Suisse de Zoology, vol. hors ser. 1: 597-610.

Scharff, N.; Coddington, J.A.; Griswold, C.E.; Hormiga, G.; Bjørn, Per de Place. 2003. When to quit? Estimating spider species richness in a northern European deciduos forest. Journal of Arachnology, 31: 246-273.

Toti, D.S.; Coyle, F.A.; Miller, J.A. 2000. A structured inventory of Appalachian grass bald and heath bald spider assemblages and a test of species richness estimator performance. Journal of Arachnology, 28: 329-345.

Walther, B.A.; Moore, J.L. 2005. The concepts of bias, precision and accuracy, and their use in testing the performance of species richness estimators, with a literature review of estimator performance. Ecography, 28: 815-829.

Wolda, H. 1981. Similarity indices, sample size and diversity. Oecologia, 50: 296-302.

Recebido em 19/02/2008

Aceito em 16/06/2009 